WORLD CONGRESS ON OSTEOPOROSIS, OSTEOARTHRITIS AND MUSCULOSKELETAL DISEASES

\title{
VIRTUAL CONGRESS
} August 26-28, 2021
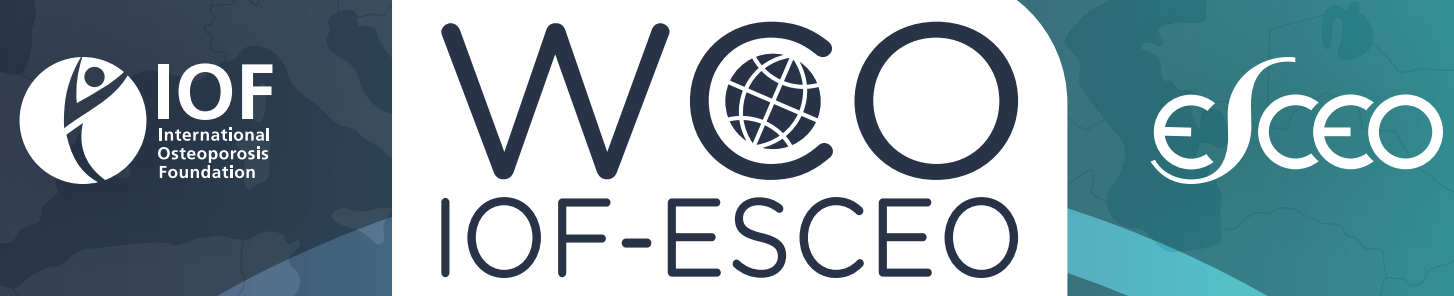

\section{VIRTUAL}

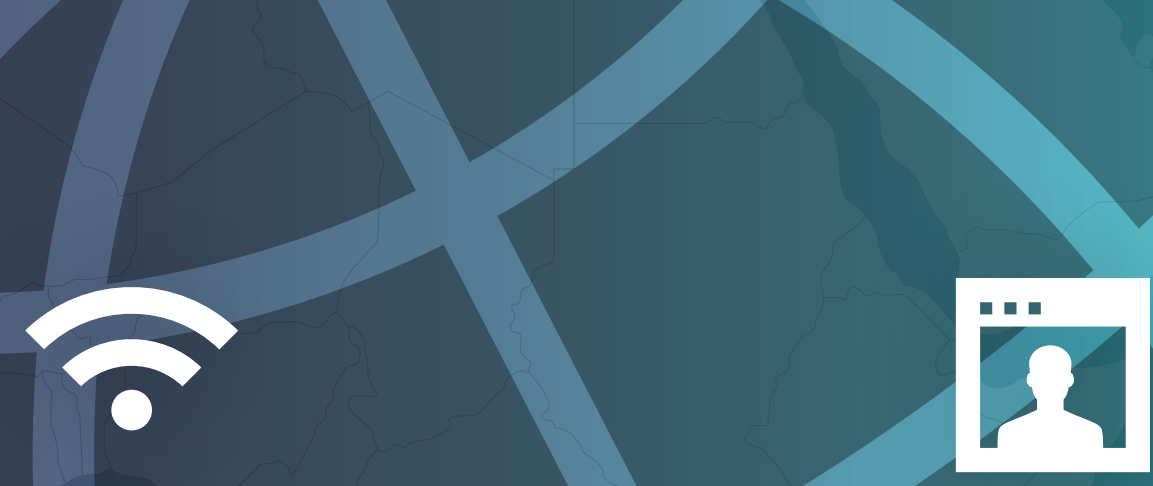


P517

EFFECT OF A MULTICOMPONENT MEDICAL

REHABILITATION PROGRAM ON PAIN AND

FATIGUE IN PATIENTS WITH OSTEOARTHRITIS AND

RHEUMATOID ARTHRITIS

N. V. Aleksandrova ${ }^{1}$, A. V. Aleksandrov ${ }^{1}$, V. A.

Aleksandrov'2, M. V. Nikitin ${ }^{3}$, R. A. Grekhov ${ }^{1}$

${ }^{1}$ Research Institute of Clinical and Experimental Rheumatology A.B. Zborovsky, Volgograd, '²olgograd State Medical University, Dept. of Hospital Therapy, Volgograd, ${ }^{3}$ Sanatorium-Resort Complex "Vulan" - Scientific and Clinical Branch of the FSB Institution "National Medical Research Center for Rehabilitation and Balneology", Gelendzhik, Russia

Objective: To evaluate the short-term effectiveness of a multicomponent rehabilitation program based on aerobic exercises in patients with osteoarthritis (OA) and rheumatoid arthritis (RA).

Methods: We observed 60 patients with OA (mean age 57.4 [49.3:71.1] y, duration of disease 14.8 [5.5:21.9] y) and 74 patients with RA (mean age $52.1 \pm 9.6 \mathrm{y}$, duration of disease 9.2 $[4.8 ; 13.6] \mathrm{y})$. The following rehabilitation technologies (RT) were used at the sanatorium-resort stage of medical rehabilitation (within $21 \mathrm{~d}$ ): I - kinesitherapy (dosed walking from 30-60 min daily), II - hydrokinesitherapy (therapeutic swimming in a pool with seawater, $10-12$ procedures for $30 \mathrm{~min}$ daily or every other day), III - low-frequency magnetotherapy (LFM) (from $0.3-100 \mathrm{~Hz}$; up to $5 \mathrm{mT}$; 10 procedures for 30 min every other day), IV - a method of functional biocontrol with biofeedback (BFB therapy) (10-12 sessions of thermal and myographic BFB therapy, for 20-25 min daily). The intensity of pain in the affected joints (while walking) was assessed using the visual analogue pain scale (VAS Huskisson). The AS $_{0-100}$ and Bristol Rheumatoid Arthritis Fatigue Numerical Rating Scales (BRAF-NRS V2) were used to assess fatigue.

Results: The combined use of RT-I and RT-II in OA patients $(n=24)$ resulted in a decrease in pain intensity $(p=0.041)$ and fatigue on the VAS ${ }_{0-100}$ scale $(p=0.044)$, and in RA patients $(n=28)$ resulted in decreased pain on the VAS scale $(p=0.031)$, decreased fatigue on the $\operatorname{VAS}_{0-100}(p=0.04)$, NRS severity and NRS-overcoming scales $(p=0.008$ and $p=0.013$, respectively). The combined use of RT-III and RT-IV in OA patients $(n=20)$ reduced pain $(p=0.017)$ and the number of swollen joints $(p=0.044)$, and in RA patients $(n=22)$ reduced NRS-overcoming $(p=0.027)$. The use of a multicomponent treatment regimen (combination of all RTs) in the group of OA patients $(n=16)$ showed a significant decrease in pain intensity $(p=0.022), V_{0-100}$ fatigue $(p=0.021)$, number of painful joints $(p=0,034)$, and in the group of patients with RA $(n=24)$ resulted in a decrease in pain intensity on the VAS scale $(p=0.018)$, number of painful joints $(p=0.023)$, level of chronic fatigue on VAS $(p=0.002)$ and all components of BRAF-NRS (NRS-degree of severity, $\mathrm{p}=0.009$ NRS-overcoming, $\mathrm{p}<0.001$; NRS-effect, $\mathrm{p}=0.033$ ).
Conclusion: Dynamics of joint syndrome indexes, pain and chronic fatigue demonstrate the positive result of multicomponent treatment scheme on the basis of combination of aerobic exercises and methods of modern physiotherapy in patients with RA. It is possible to achieve significant changes in the studied parameters in patients with $\mathrm{OA}$ even with the isolated use of aerobic exercises (walking, swimming).

P518

GENETIC VARIATION IN VITAMIN D METABOLISM IN THE MOTHER, RATHER THAN THE FETUS, IS ASSOCIATED WITH UMBILICAL CORD BLOOD 25-HYDROXYVITAMIN D STATUS: FINDINGS FROM THE MAVIDOS RANDOMIZED CONTROLLED TRIAL R. J. Moon', S. D'Angelo', E. M. Curtis'1, L. Cooke'2, S. R. Crozier', K. M. Godfrey', N. J. Graham², J. W. Holloway'2, R. Lewis $^{2}$, J. Cleal' ${ }^{2}$ J. H. Davies ${ }^{3}$, H. M. Inskip ${ }^{1}$, C. Cooper', N. C. Harvey ${ }^{1}$

${ }^{1}$ MRC Lifecourse Epidemiology Unit, University of Southampton, ${ }^{2}$ Human Health \& Development, Faculty of Medicine, University of Southampton, ${ }^{3}$ Paediatric Endocrinology, University Hospital Southampton NHS Foundation Trust, Southampton, UK

Objective: Single nucleotide polymorphisms (SNP) in the vitamin $D$ metabolism pathway are associated with serum 25-hydroxyvita$\min \mathrm{D}[25(\mathrm{OH}) \mathrm{D}]$ in adulthood. The fetus is dependent on transfer of $25(\mathrm{OH}) \mathrm{D}$ across the placenta. We assessed whether maternal and/or offspring SNPs in this pathway are associated with umbilical cord blood (UCB) 25(OH)D, and if antenatal vitamin D supplementation modifies the relationships.

Methods: MAVIDOS is a randomised placebo controlled trial of $1000 \mathrm{IU} / \mathrm{d}$ cholecalciferol from 14 weeks gestation until delivery. UCB $25(\mathrm{OH}) \mathrm{D}$ was assessed by tandem mass spectrometry. SNPs at rs12785878 (7-dehydrocholesterol reductase, DHCR7), rs10741657 (25-hydroxylase, CYP2R1), rs6013897 (24-hydroxylase, CYP24A1) and rs2282679 (vitamin D binding protein [DBP], $G C$ ) were genotyped in maternal and UCB blood. Associations between deseasonalised 25(OH)D generated by Fourier analysis and the SNPs were assessed by linear regression for each study group using an additive model ( $\beta=$ change in $25(\mathrm{OH}) \mathrm{D}$ per additional common allele). Models were adjusted for maternal age, triceps skinfold thickness and late pregnancy smoking as these were associated with UCB 25(OH)D.

Results: 329 mother-offspring pairs were included (165 cholecalciferol, 185 placebo). The cholecalciferol group had higher UCB $25(\mathrm{OH}) \mathrm{D}$ (mean $42.3 \mathrm{nmol} / \mathrm{l} ; \mathrm{SD} 13.1$ ) than the placebo group (mean $28.6 \mathrm{nmol} / \mathrm{l} ;$ SD 12.1), $\mathrm{p}<0.001$. The SNP at rs22825679 (GC) in the mother was associated with UCB $25(\mathrm{OH}) \mathrm{D}$ in the cholecalciferol group ( $\beta=4.2 \mathrm{nmol} / \mathrm{l}$ per allele; $95 \% \mathrm{Cl} 1.0,7.3)$. The effect of this SNP in the placebo group was smaller $(\beta=1.9$ 